\title{
STAR COLORING OUTERPLANAR BIPARTITE GRAPHS
}

\author{
Radhika Ramamurthi and Gina Sanders \\ Department of Mathematics \\ California State University San Marcos \\ San Marcos, CA 92096-0001 USA \\ e-mail: gsanders@palomar.edu
}

\begin{abstract}
A proper coloring of the vertices of a graph is called a star coloring if at least three colors are used on every 4 -vertex path. We show that all outerplanar bipartite graphs can be star colored using only five colors and construct the smallest known example that requires five colors.
\end{abstract}

Keywords: chromatic number, star coloring, outerplanar bipartite graph.

2010 Mathematics Subject Classification: 05C15.

\section{REFERENCES}

[1] M.O. Albertson, G.C. Chappell, H.A. Kierstead, A. Kündgen and R. Ramamurthi, Coloring with no 2-colored $P_{4}$ 's, Electron. J. Combin. 11 (2004) \#R26.

[2] O. V. Borodin, On acyclic colorings of planar graphs, Discrete Math. 25 (1979) 211236. doi:10.1016/0012-365X(79)90077-3

[3] M. Chen, A. Raspaud and W. Wang, 6-star-coloring of subcubic graphs, J. Graph Theory 72 (2013) 128-145. doi:10.1002/jgt.21636

[4] G. Fertin, A. Raspaud and B. Reed, Star coloring of graphs, J. Graph Theory 47 (2004) $163-182$. doi:10.1002/jgt.20029

[5] B. Grünbaum, Acyclic colorings of planar graphs, Israel J. Math. 14 (1973) 390408. doi:10.1007/BF02764716

[6] H.A. Kierstead, A. Kündgen and C. Timmons, Star coloring bipartite planar graphs, J. Graph Theory 60 (2009) 1-10. doi:10.1002/jgt.20342 
[7] J. Nešetřil and P. Ossona de Mendez, Colorings and homomorphisms of minor closed classes, in: B. Aronov, S. Basu, J. Pach and M. Sharir (Eds.), Discrete and Computational Geometry: The Goodman-Pollack Festschrift (Springer-Verlag, Berlin, 2003) 651-664.

doi:10.1007/978-3-642-55566-4

[8] G. Sanders, Star Coloring of Outerplanar Bipartite Graphs, M.Sc. Thesis (California State University San Marcos, 2005).

[9] C. Timmons, Star Coloring Planar Graphs, M.Sc. Thesis (California State University San Marcos, 2007).

[10] D.B. West, Introduction to Graph Theory, Second Edition (Prentice Hall, 2001).

Received 7 August 2014

Revised 19 December 2017

Accepted 20 December 2017 